Laura Tabuenca Del Barrio

Marcos Mozo Cuadrado

Enrique Borque Rodríguez-

Maimón

Alicia Zubicoa Eneriz

Andrés Garralda Luquín

\section{Decreased painful visual acuity. Corynebacterium macginleyi blebitis-endophthalmitis infection}

Complejo Hospitalario de Navarra, Ophthalmology. Pamplona (Navarra). Spain

\section{Article history}

Received: 18 September 2019; Revision Requested: 14 October 2019; Revision Received: 27 October 2019; Accepted: 31 October 2019; Published: 8 January 2020

\section{Sir,}

A 74 year-old female presented with pain and blurred vision in her left eye since one day. As a personal records included bilateral chronic open angle glaucoma, bilateral trabeculectomy and cataract surgery in both eyes. Visual acuity (VA) was 20/40 in the right eye and light perception in the left eye. Slit-lamp examination showed a white conjunctival swelling superiorly (figure 1A) and hypopyon in anterior chamber. Fundus examination revealed a dense vitritis. Applanation intraocular pressure was $4 \mathrm{mmHg}$. Echography manifested echogenic shadows in the vitreous cavity (figure 1B). A culture of conjunctival swelling was performed and revealed Corynebacterium macginleyi growth. The material was cultured on Columbia agar plates supplemented with $5 \%$ sheep blood and chocolate agar for $24 \mathrm{~h}$ at $37^{\circ} \mathrm{C}$ in a $5 \% \mathrm{CO}_{2}$-enriched atmosphere and on MacConkey agar at $37^{\circ} \mathrm{C}$ in ambient air. The lipophilic Corynebacterium were identified by API Coryne system, in conjunction with API Coryne database. The antibiogram showed susceptibility to ciprofloxacin, chloramphenicol, gentamicin, tobramycin, neomycin and tetracyclines. The patient was diagnosis of infectious bleb-associated endophthalmitis in left eye. The treatment involved ceftazidime $(2.25 \mathrm{mg} / 0.1 \mathrm{ml})$ and vancomycin (1 $\mathrm{mg} / 0.1 \mathrm{ml}$ ) intravitreal injections. In addition, intensive topical antibiotics of ceftazidime and vancomycin together with topical (1 drop/8 hours) and oral (400 mg/12h) ciprofloxacin were administered. Forty-eight hours later, intravitreal injections were repeated keeping the same topical and oral treatment. The treatment was well tolerated and the pain was subsiding. One week later, when anterior pole allowed a good visualization, a washing anterior chamber and a pars plana vitrectomy were carried out. Oral ciprofloxacin was suspended and topical

Correspondence:

Laura Tabuenca Del Barrio

Complejo Hospitalario de Navarra, Ophthalmology.

Calle Irunlarrea s/n, 31008 Pamplona (Navarra). Spain

Phone: +34675621164

E-mail:I.tabuenca.del@navarra.es tobramycin, dexamethasone and ciprofloxacin (1 drop/8 hours) were instilled for one month. Finally, VA was 20/100 and a fibrous bleb was observed (figure 2).

Glaucoma is a pathology which requires surgical techniques in some patients. The trabeculectomy involves a defect in the sclera and a filtering conjunctival bleb to allow excess aqueous humour leaves out of the anterior chamber. The finality of this surgery is a reduction of intraocular pressure in patients with a medical management failure and intracameral cefuroxime is used as antibiotic prophylaxis in surgical procedure.

Blebitis with endophthalmitis is an infrequent complication after glaucoma filtering surgery which consists of an infected conjunctival bleb with anterior chamber and vitreous affection. Typically occurs months to years following surgery and usually has a devastating prognosis.

C. macginleyi is an infrequent pathogen of blebitis. To the best of our knowledge, this is the first case report of a $C$. macginleyi blebitis-endophthalmitis infection and its correct treatment is related to prevent subsequent complications.

Blebitis may be associated or not with vitreous infection. The onset is usually abrupt in cases which involve endophthalmitis: the patients presents with sudden onset of eye pain, decreased vision and red purulence bleb. This affection may be classificated in three ways: isolated blebitis, early bleb-associated endophthalmitis (BAE) or late bleb-associated endophthalmitis, which have different pathogenesis and prognosis [1].

Staphylococcus epidermidis and Staphylococcus aureus are the most common cause in isolated blebitis. Early BAE appears in the first month after surgery and $S$. epidermidis is the main organism implicate. Generally, it is caused by the perioperative introduction of host flora. In late BAE, that appears after one month of surgery action, Streptococcus species, Haemophilus influenzae or Moraxella catarrhalis are the main cause migrating through thin-walled blebs and generating a poor outcome [1]. 


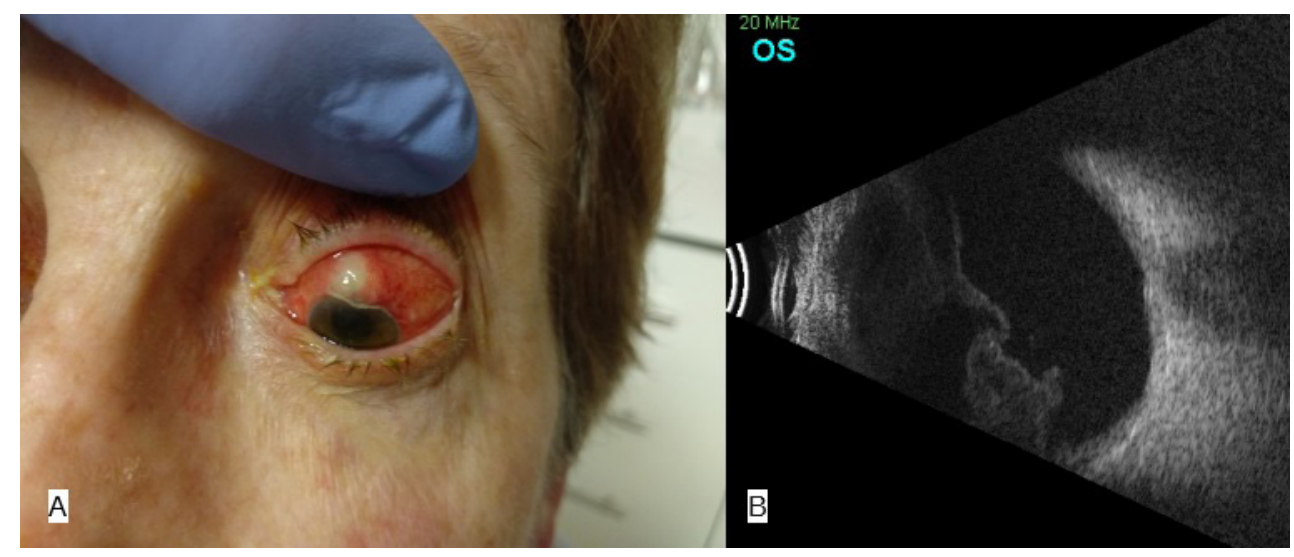

Figure $1 \quad$ A red and purulence superior conjunctival bleb (A). Echography: Hyperechogenic shadows demonstrate dense vitritis (B)

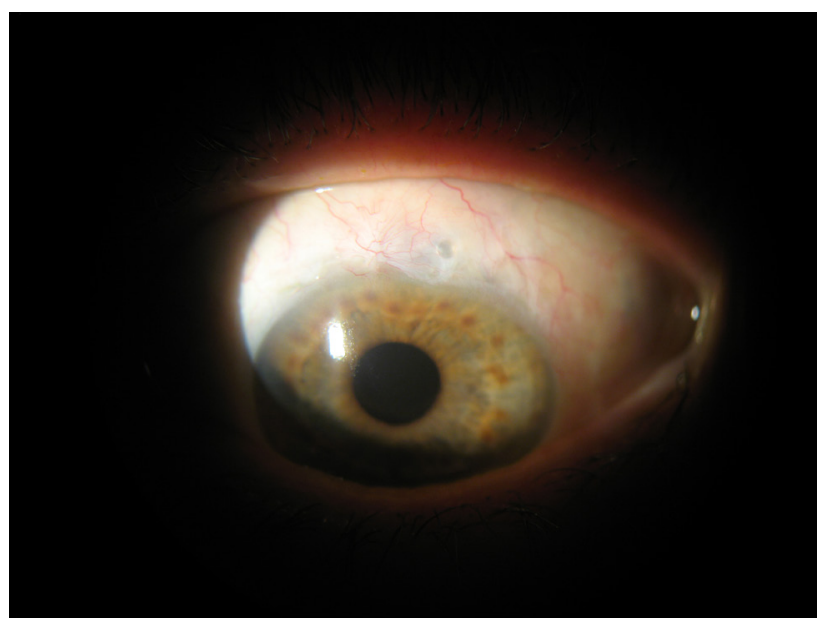

\begin{tabular}{l|l} 
Figure 2 & Slit-lamp photograph. Ocular surface
\end{tabular} after medical and surgical therapy. A fibrous conjunctival bleb is observed.

Our clinical case appears two years after surgical technique, so it is a late BAE.

C. macginleyi is a lipophilic dipheroidal conjunctival Corynebacteria. It is usually a habitual resident of conjunctival flora and mainly generates conjunctivitis [2-4]. It is unsurprising that Corynebacteria generates blebitis due to the implication of conjunctival tissue in filtering glaucoma surgery [2]. In spite of this, it is the first time that this organism affects anterior chamber and vitreous too. An infected bleb culture must be obtained in all the patients.

C. macginleyi was described by Riegel et al. in the study of lipophilic Corynebacterium [5]. The identification of C. macginleyi is based on biochemical test and API Coryne (bioMèrieux) system in conjunction with API Coryne database. The results consist of positive nitrate reduction, positive alkaline phosphatase, negative pyrazinaminidase, acid production from glucose and sucrose but not from maltose [6].
A susceptibility antibiotic testing should be performed: it is usually susceptible to beta-lactams, tetracyclines, fusidic acid, glycopeptides and rifampicin [2]. Funke et al. described the susceptibility to antibiotics of $C$. macginleyi finding very low minimum inhibitory concentration values for almost all tested antibiotics, except macrolides [3].

An intravitreal vancomycin $(1 \mathrm{mg} / 0.1 \mathrm{ml})$ plus ceftazidime $(2.25 \mathrm{mg} / 0.1 \mathrm{ml})$ therapy is indicated in BAE. If vitreous inflammation persists, intravitreal injections may be repeated after 48 hours in the same doses and vitrectomy should be considered [7]. In our case, a favourable response with painful decreased was observed after antibiotic treatment beginning but vitrectomy was necessary to eliminate vitreous infection consequences.

The prognosis in late BAE is poor, but in our case report the large antibiotic susceptibility of $C$. macginleyi could implicate a better outcome obtaining a 20/100 final visual acuity instead of a total loss of vision.

\section{FUNDING}

None to declare.

\section{CONFLICTS OF INTEREST}

The authors declare that they have no conflicts of interest

\section{REFERENCES}

1. Navin Prasad, MD, Mark A, Latina, MD. Blebitis and endophthalmitis after glaucoma filtering surgery. Int Ophthalmology Clin. 2007; 47 (2): 85-97. doi:10.1097/II0.0b013e31803775c6

2. Qin $V_{1}$ Laurent $T$, Ledoux A. - associated blebitis: a case report. J Glaucoma. 2018; 27 (10): 174 - 176. doi: 10.1097| IJG.0000000000001051

3. Funke $G$, Pagano-Niederer $M$, Bernauer W. Corynebacterium 
macginleyi has been isolated exclusively from conjunctival swabs. J Clin Microbiol. 1998; 36 (12): 3670-3673. PMID: 9817893

4. Ortiz de la Tabla V, Martín C, Martínez C. Conjuntivitis por Enf Infecc Microbiol Clin. 2000. 18(9):481-2. PMID: 11149176

5. Riegel P, Ruimy $R$, de Briel $D$, Prévost $G$, Jehl F, Christen $R$ et al. Genomic diversity and phylogenetic relationship among lipid-requiring diphtheroides from humans and characterization of sp. Int J Syst Bacteriol. 1995; 45(1): 128-133. doi: 10.1099/00207713-45$1-128$

6. Joussen MA, Funke G, Joussen F. Georg Herbertz. : a conjunctiva specific pathogen. Br J Ophthalmol. 2000; 84: 1420 - 1422. PMID: 11090486

7. Marlene L Durand, MD. Bacterial endophthalmitis. UpToDate. https://www-uptodate-com.na-cdib.a17.csinet.es/contents/ bacterial-endophthalmitis?search=bacterial\%20endophthalmitis\&tsource=search_resultttselectedTitle=1 24\&usage_type=defaultetdisplay_rank=1. 2018 (accesed 5th August 2019). 